\title{
Editorial
}

\section{Mathematical Methods and Modeling in Machine Fault Diagnosis}

\author{
Ruqiang Yan, ${ }^{1}$ Xuefeng Chen, ${ }^{2}$ Weihua $\mathrm{Li}^{3}$ and Shuangwen Sheng ${ }^{4}$ \\ ${ }^{1}$ School of Instrument Science and Engineering, Southeast University, Nanjing 210096, China \\ ${ }^{2}$ School of Mechanical Engineering, Xian Jiaotong University, Xian 710049, China \\ ${ }^{3}$ School of Mechanical and Automotive Engineering, South China University of Technology, Guangzhou 510640, China \\ ${ }^{4}$ National Renewable Energy Laboratory, Golden, CO 80401, USA
}

Correspondence should be addressed to Ruqiang Yan; ruqiang@seu.edu.cn

Received 20 August 2014; Accepted 20 August 2014; Published 18 December 2014

Copyright (C) 2014 Ruqiang Yan et al. This is an open access article distributed under the Creative Commons Attribution License, which permits unrestricted use, distribution, and reproduction in any medium, provided the original work is properly cited.

Modern mathematics has often been utilized as an effective tool to model mechanical equipment so that their dynamic characteristics can be studied analytically. This will help identify potential failures of mechanical equipment by observing change in the equipment's dynamic parameters. On the other hand, dynamic signals are also important and provide reliable information about the equipment's working status. Modern mathematics has also provided us with a systematic way to design and implement various signal processing methods, which are used to analyze these dynamic signals, and to enhance intrinsic signal components that are directly related to machine failures. This special issue is aimed at stimulating not only new insights on mathematical methods for modeling but also recently developed signal processing methods, such as sparse decomposition with potential applications in machine fault diagnosis. The papers included in this special issue provide a glimpse into some of the research and applications in the field of machine fault diagnosis through applications of the modern mathematical methods.

Manufacturing systems are often operated under high pressure, at high temperatures, with fast-flowing material and complex mechanisms. Progressive faults can be caused by deterioration of the operating environment or aging and show stochastic properties. X. Gong et al. explored an adaptive maintenance model to detect progressive faults. The model is used to monitor the environmental states of the systems and compares the inputs/outputs and presettings to detect faults. Experiments performed on the process environment of an air separation plant verified the effectiveness of the maintenance model.

Rotating machines and/or machine components have been widely used in modern factories. Timely monitoring and diagnosing their working conditions is critical to avoid possible losses of production due to machine breakdown, as these machines are always running under varying speeds and loading conditions, which make the vibration signal more complicated. To address this issue, S. Zhang and W. Li explored an unsupervised neighborhood preserving embedding (NPE) algorithm with application to bearing fault diagnosis. Their study indicates the NPE is capable of extracting the most discriminative features for classification. Furthermore, when combined with a self-organizing map, the bearing degradation process can be assessed accurately. F. Wang et al. proposed a new tensor manifold method to realize the bearing fault feature extraction. The time-frequency characteristics of the signals are extracted using the tensor manifold. The proposed method can reduce the information redundancy and information loss and effectively distinguish different bearing fault states. Q. He et al. acquired sensitive features through a combination of local discriminant bases (LDB) and locality preserving projections. The proposed feature extraction method combines the merits of these two techniques and extracts the inherent pattern structure embedded in the discriminatory features. The new feature 
not only considers the static discriminatory wavelet packet node features themselves, but also considers the dynamic sensitive class pattern structure embedded in the samples. The proposed feature displays valuable benefits for data classification, and its effectiveness is verified by case studies on vibration data-based classification of bearing fault types and severities. W. Cheng et al. reported a feature selection criterion, mean impact variance (MIVAR), which can be used to determine which feature is more suitable for the artificial neural network-based bearing fault classification. The MIVAR values of all the features are calculated by changing the input vectors and then measuring the differences of the output vectors after the training process of the back propagation neural network. It is proved that using the features with higher MIVAR values can lead to a higher recognition rate and the corresponding performance is as good as that of a traditional feature selection algorithm, such as the principal component analysis (PCA) algorithm. In another study, $\mathrm{H}$. Wang et al. developed a new kind of peakbased strategy to enhance the weak bearing fault detection. A peak-based piecewise recombination is proposed to convert middle frequency components into low frequency ones; then the vibration signal becomes so smooth that its sparseness in the wavelet domain will improve significantly. This helps eliminate interference noise and detect weak bearing faults. H. Zhang et al. proposed a new stochastic resonance (SR) model for bearing fault diagnosis. The new SR model has a joint Woods-Saxon and Gaussian potential whose parameters are not coupled and thus easily tuned to optimize the output signal-to-noise ratio (SNR). In addition, a smoother potential bottom and a steeper potential wall lead to stable particle motion within each potential well and thus avoid the unexpected noise. The novel bistable SR model is verified to be capable of offering a higher output SNR and a wider bandwidth in weak signal detection. S. Dong et al. presented a hybrid method to solve the rotating machinery fault diagnosis problem, which is based on PCA to extract the characteristic features and the Morlet kernel support vector machine to achieve the fault classification. The proposed method makes good use of the advantage of all parts together to obtain better recognition accuracy. $H$. Sun et al. studied a fault diagnosis method for rotating machinery based on a multiwaveletadaptive threshold denoising and mutation particle swarm optimization algorithm (MPSO). A Geronimo, Hardin, and Massopust (GHM) multiwavelet was employed for extracting weak fault features under background noise, and the method of adaptively selecting the appropriate threshold for the multiwavelet with an energy ratio of a multiwavelet coefficient was presented. An MPSO algorithm with an adaptive inertia weight adjustment and a particle mutation was proposed for condition identification. Practical examples of fault diagnosis for rolling element bearings verified the effectiveness of the proposed method. W. Fan et al. proposed a novel method for machinery fault diagnosis by combining the wavelet basis and majorization-minimization algorithm. With the proposed method, transients hidden in the noisy signal can be converted into sparse coefficients; thus the transients can be detected sparsely. The effectiveness of the proposed method is verified by both simulated and measured gearbox vibration signals. Results show that the proposed method outperforms the method based on split-augmented Lagrangian shrinkage algorithm in convergence and detection effect.

The aeroengine is one of the key components in an aircraft and its reliability directly affects flight safety. Rub-impact caused by decreased clearance between the rotor and stator in an aeroengine generates unexpected vibrations, making the aeroengine not function well and even causing catastrophic consequences. Identifying the rub-impact fault at its early stage in an aeroengine becomes a critical task. J. Cui et al. developed an integrated approach, based on the improved LDB and support vector machine, for aeroengine fault diagnosis. The experimental results verified that the developed approach is able to classify different aeroengine working conditions. Predicting fatigue crack propagation of aircraft components under service loading is necessary to ensure flight safety. S. Jiang et al. developed a theoretical model to predict fatigue crack growth behavior under the single overload, in which crack closure and a plastic zone concept are considered. The model was validated in D16 aluminum alloy and 350WT steel subjected to several different loading spectra, and the predictions matched experimental data well.

The blade is a key component of the centrifugal compressor. However, crack and fatigue failure can often occur as the blade is subjected to centrifugal forces, gas pressure, friction force, and so on. Thus, it is important to have an early warning for blade cracking. $\mathrm{H}$. Li et al. used pressure pulsation information to diagnose blade crack from a blade vibration transfer process analysis. A dynamic strain sensor is installed on the blade to determine the crack characteristic frequency. The results show that this method can be helpful for blade crack classification in centrifugal compressors.

Rail track inspection and maintenance are key factors in keeping trains operating safely. J. Zhao et al. presented a two-dimensional impact reconstruction method to perform online inspection of rails and to find defects. The method utilizes preprocessing technology to convert time domain vertical vibration signals, acquired by a wireless sensor network, to space signals. The modern time frequency analysis method is improved to reconstruct the obtained multisensor information. Then, image fusion processing technology, based on spectrum threshold processing and node color labeling, is introduced to reduce the noise, blank the periodic impact signals caused by rail joints and locomotive running gear, and convert the aperiodic impact signals caused by rail defects to partial periodic impact signals. This method can be used to do an online analysis of the vertical vibration signals of a train when it is running, extract the aperiodic impact features caused by the rail defects, perform the online inspection, and locate rail defects.

Fiber optic current transducers (FOCTs) are the basic components of power systems. To ensure low failure and high reliability of FOCTs, it is vital to study methods of condition monitoring and fault diagnosis in such transducers. As a complement to the frequency domain analysis, L. Wang et al. analyzed time domain and frequency domain features of fiber optic current transformers' measurement data, established correspondence between the physical characteristics of key components in transformer and data features, and then built 
a diagnostic analysis model based on Allan variance. The fiber optic current transformer's health state can be determined from the Allan variance calculation results.

Magnetic flux leakage (MFL) is an efficient method for detecting pipeline flaws. $\mathrm{Z}$. Wu et al. proposed a new adaptive channel equalization approach for processing a MFL signal prior to flaw characterization. The approach performs channel equalization by using single layer neural networks, and the fast-learning algorithm of an extreme learning machine is used to achieve excellent processing speed. Focusing on the signal of a flaw, a PCA-based flaw detecting algorithm is given to locate the flaw signal, which is verified from both theoretical and simulation studies.

Polymer matrix composite materials have been developed rapidly to meet the demands for better materials with higher standards of performance and reliability in structures and machines. X. Zhu et al. employed a viscoplastic constitutive model in the micromechanical method, based on a generalized model of cells, to analyze the inelastic, rate dependent stress-strain response of fiber-reinforced polymer matrix composites with three different microstructures at different fiber off-axis angle conditions. Acceptable agreement is observed between the model predictions and experimental results found in the literature. The results show that the stress-strain curves are sensitive to the strain rate and the microstructure parameters play an important role in the behavior of a polymer matrix.

\section{Acknowledgments}

As our editorial work comes to an end, we would like to express our deep appreciation to all the authors who supported this special issue by contributing papers. We are also grateful to all the reviewers for their insightful and constructive comments.

Ruqiang Yan

Xuefeng Chen

Weihua Li

Shuangwen Sheng 


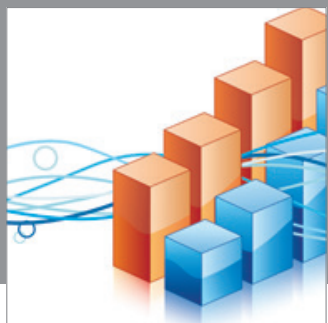

Advances in

Operations Research

mansans

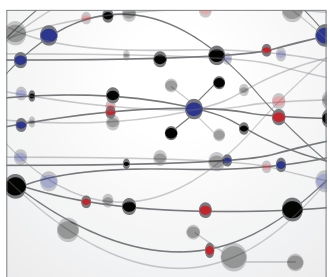

The Scientific World Journal
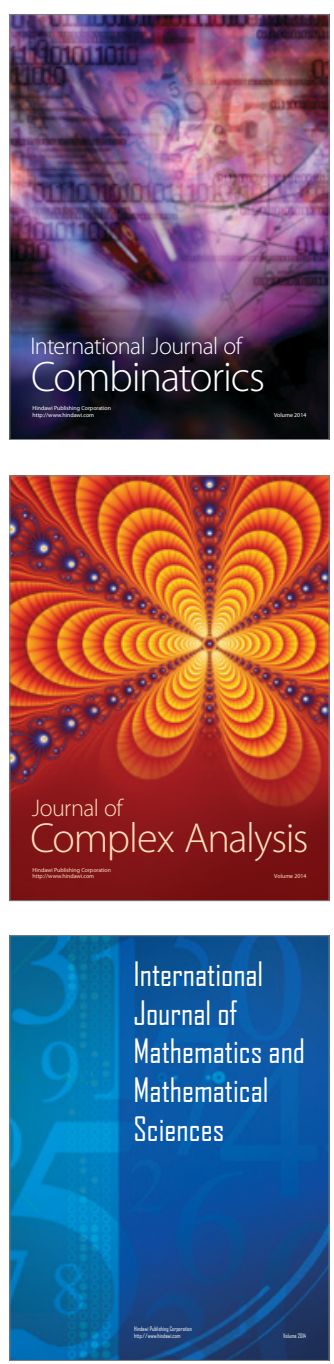
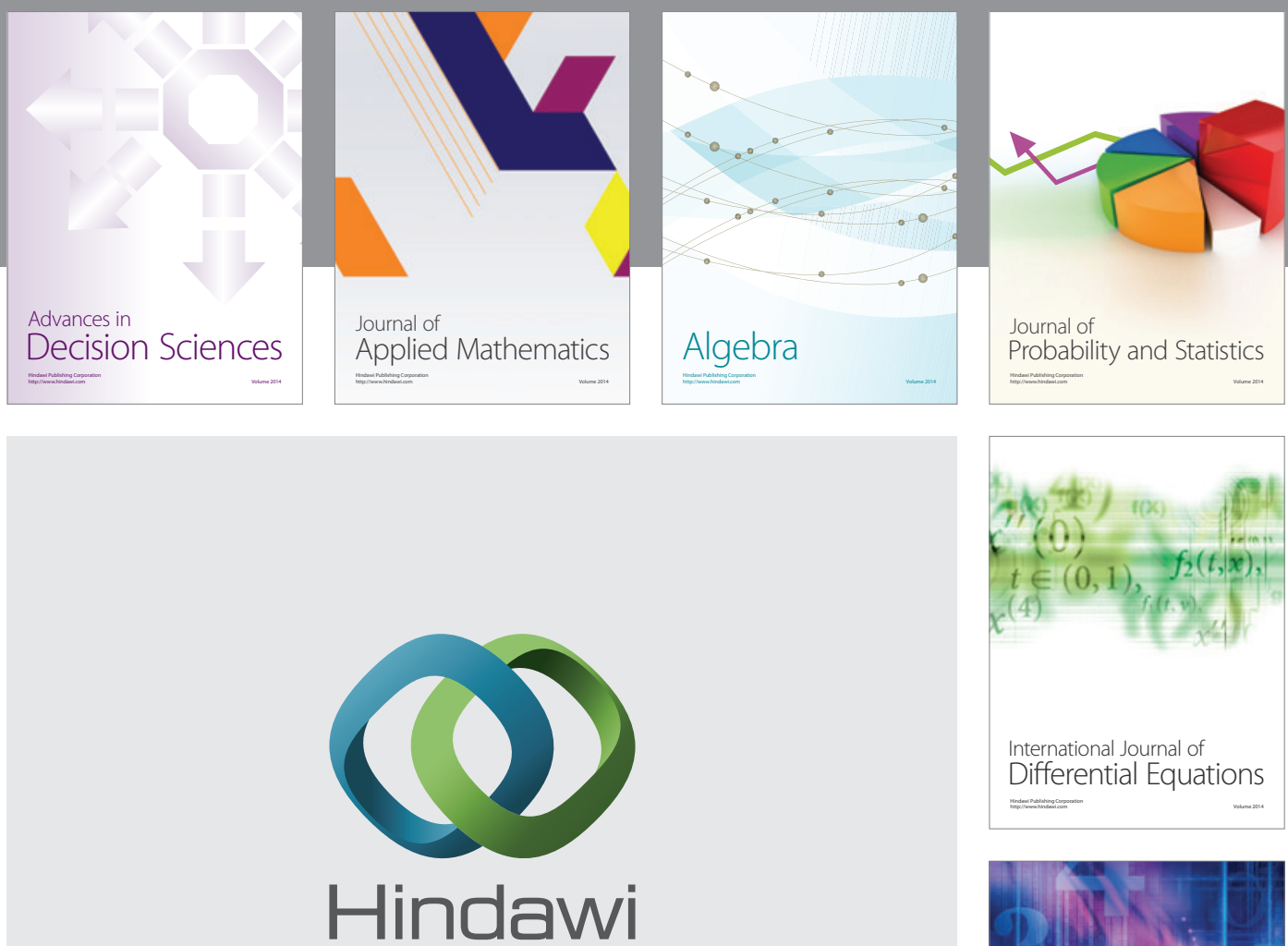

Submit your manuscripts at http://www.hindawi.com
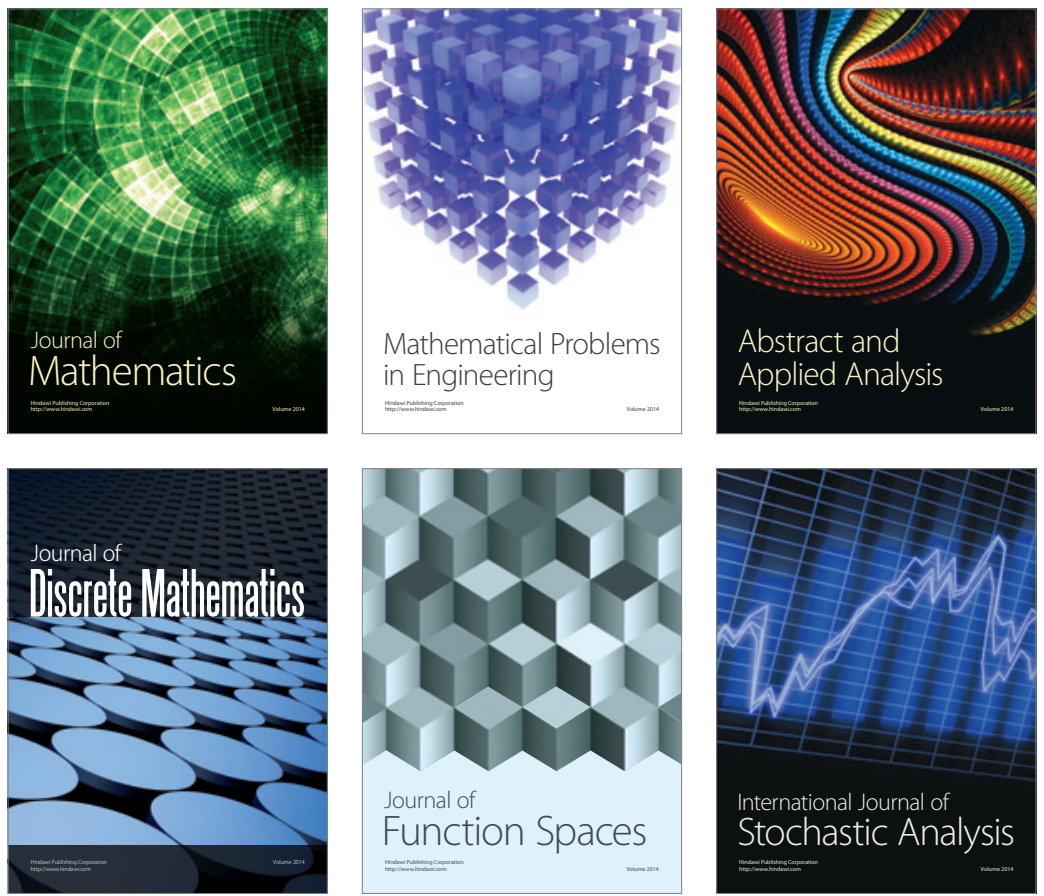

Journal of

Function Spaces

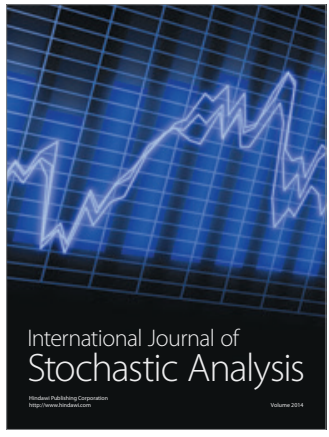

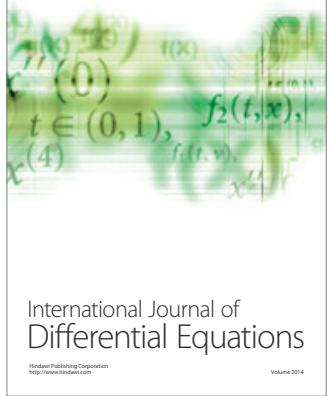
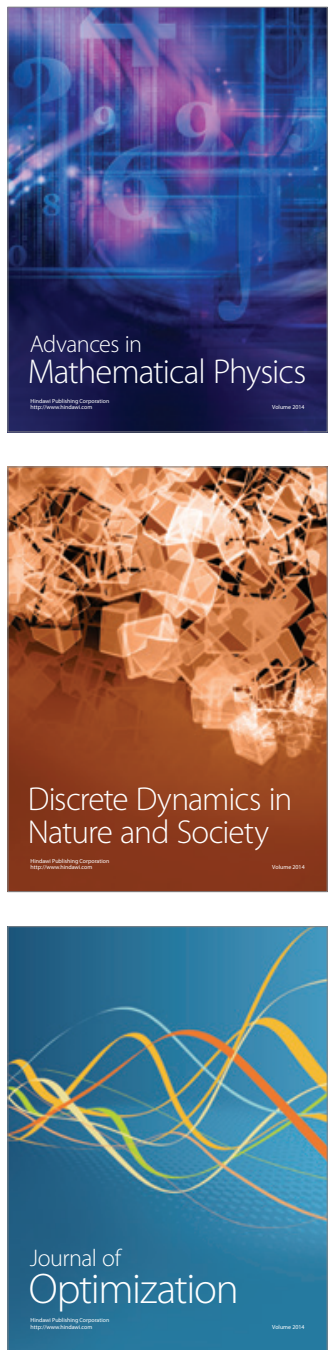